\title{
Adsorptive molecularly imprinted composite membranes for chiral separation of phenylalanine
}

\author{
Nasrullah Shah ${ }^{1,2^{*}}$, Touseef Rehan ${ }^{3}$, Joong Kon Park ${ }^{1^{*}}$ \\ ${ }^{1}$ Kyungpook National University, Department of Chemical Engineering, Taegu, Korea \\ ${ }^{2}$ Abdul Wali Khan University Mardan, Department of Chemistry, Mardan, Pakistan \\ ${ }^{3}$ Quaid-i-Azam University, Department of Biochemistry, Islamabad, Pakistan \\ "Corresponding authors: e-mail: nasrullah@awkum.edu.pk, parkjk@knu.ac.kr
}

\begin{abstract}
Two types of composite imprinted membranes, i.e., composite membrane comprised of D-Phe imprinted beads and D-Phe imprinted membrane or DCM and composite membrane comprised of L-Phe imprinted beads and L-Phe imprinted membranes or LCM, were synthesized by phase inversion technique after a uniform dispersion of beads within the polymeric solutions using simple physico-mechanical process. The assemblies of the prepared DCM, LCM and control membranes were employed in ultrafiltration for chiral separation of D, L-Phenylalanine racemate solution. DCM and LCM showed an improved adsorption capacity $\left(0.334 \mathrm{mg} \mathrm{g}^{-1}\right.$ and $0.365 \mathrm{mg} \mathrm{g}^{-1} \mathrm{re}^{-}$ spectively), and adsorption selectivity (2.72 and 2.98 respectively). However, the percent rejection of the template and counter enantiomer were lower than that of control membranes. Compared to control membrane, the DCM and LCM showed inverse permselectivity. These composite membranes having better adsorption and separation ability for Phenylalanine racemate solution will be suitable in the future for various other applications.
\end{abstract}

Keywords: molecularly imprinted composite membranes, Phe imprinted beads, Phe imprinted membranes; ultrafiltration, adsorption selectivity.

\section{INTRODUCTION}

Molecular imprinting is one of the extensive research approaches for preparing a novel type of adsorbing material with recognition sites specific for desired substances ${ }^{1-3}$. The use of molecularly imprinted membranes (MIMs) in ultrafiltration can be considered better than other separation processes in a sense that they have high adsorption capacity due to a large surface area, faster transport of substrate molecules and faster adsorption process due to faster occupation of the binding $\operatorname{sites}^{4-6}$. Superior stability, easily scale up and low preparation cost for most of the target analytes also make their importance known in various fields e.g. food, pharmaceutics, and the environment, etc. ${ }^{7-11}$.

Using the advantageous behavior of composite materials, MIMs may be made better in their functioning by incorporating some functional filler. That's why different attempts have been made to prepare MIP composite membrane systems using MIP beads/fibers as a reinforcing or dispersing phase for advanced molecular separation $^{12-17}$. The incorporation of MIP beads/fibers into the membranes has great influence on its performance if their binding sites remain intact after encapsulation. The incorporated MIP beads/fibers with active sites may greatly enhance the extraction, adsorption and selective separation capability of the system ${ }^{17}, 18$. The surface area and aspect ratio of the reinforcing particle and its adhesion to the matrix are of great importance, because they control the final properties of the composites. The best performance of polymeric composites is achieved only when there is no agglomeration of filler dispersed in the polymer matrix ${ }^{19}$. Similarly, the MIP micro/nano-particles prepared by either suspension or precipitation polymerization play a vital role in enhancing the binding efficiency of the MIP system due to their regular shape and large surface area ${ }^{13}$.
The two enantiomers of racemic mixture exhibit entirely different biological and pharmaceutical activities which make it necessary to separate the two. The cost and efficiency of the separation technique for the resolution of racemic mixture is important from the economic point of view ${ }^{20-23}$. The use of MIP composite membranes is suitable in aqueous medium for achieving high selective adsorption and separation of structurally similar compounds ${ }^{1,24-26}$. Due to high adsorption capacity these also have their potential applications in various fields such as concentration, fractionation, purification, sensors, drug delivery systems and in scaffolds etc. ${ }^{27-31}$

The aim of this work was to develop and study the role of novel and cost effective phenylalanine (Phe) imprinted adsorptive composite membranes with high adsorption capacity and selectivity along with maintaining the typical membrane characteristics. In the present study, development of molecularly imprinted composite membranes by incorporating the newly prepared D-Phe and L-Phe imprinted P (MMA-co-EGDMA) submicron/ nanoscale beads (DIBs and LIBs respectively) ${ }^{30}$; into the D-Phe and L-Phe imprinted membranes (DIMs and LIMs) matrix respectively, is reported. The prepared Phe imprinted composite membranes based on the selective adsorption phenomenon were employed in an ultrafiltration system to achieve high adsorption capacity and improved selectivity at a faster rate.

\section{EXPERIMENTAL}

\section{Material}

D-Phe, L-Phe, D, L-Phe racemic mixture (D, L-Phe), methacrylic acid (MAA), ethyleneglycoldimethacrylate (EGDMA), and trifluoroacetic acid (TFA) were purchased from Sigma-Aldrich (St Louis, MO, USA); 2.2-azobisisobutyronitrile (AIBN) was obtained from Junsei Chemical Co., Ltd. (Japan); toluene and dime- 
thylsulfoxide (DMSO) were the products of Duksun Pure Chemical Co., (Korea); sodium dodycyl sulfate (SDS) was from Fluka (Switzerland), acrylic acid (AA), polyvinyl alcohol (PVA), copper sulfate $\left(\mathrm{CuSO}_{4} \cdot 5 \mathrm{H}_{2} \mathrm{O}\right)$ and acrylonitrile (AN) were obtained from Yakuri Pure Chemicals Co., (Osaka, Japan). Acetonitrile was from Honeywell Int'l Inc. (USA). All reagents used were of analytical grade.

\section{Procedure for preparation of DCM and LCM}

For synthesis of molecularly imprinted composite membranes the DIBs and LIBs were prepared by modified suspension polymerization method ${ }^{20,32,33}$ and the D-Phe and L-Phe incorporated polymeric solution were prepared by in situ polymerization method ${ }^{34}$.

\section{Preparation of DIBs and LIBs}

DIBs and LIBs were prepared by mixing an organic solution of D-Phe or L-Phe (0.116 g, $1 \mathrm{mmol})$, MAA $(0.34 \mathrm{ml}, 4 \mathrm{mmol})$, acetic acid $(0.6 \mathrm{ml})$, TFA $(0.4 \mathrm{ml})$, EGDMA $(3.77 \mathrm{ml}, 20 \mathrm{mmol})$ and toluene $(3 \mathrm{ml})$, AIBN $(0.15 \mathrm{~g})$ with an aqueous solution of PVA $(3.45 \mathrm{~g} / 130 \mathrm{~mL})$ and SDS $(1.0 \mathrm{~g} / 20 \mathrm{~mL})$ in the polymerization reactor. Then the solution was purged with $\mathrm{N}_{2}$ and polymerized at $60^{\circ} \mathrm{C}$ for 24 hours. DIBs with round shape and porous surface were obtained by this method. The average beads size was in the range of $100 \mathrm{~nm} \sim 1.5 \mu \mathrm{m}$.

Preparation of D-Phe and L-Phe incorporated poly (acrylonitrile-co-acrylic acid) solution (DI- or LI-P (AN-co-AA) solution

DI- and LI-P (AN-co-AA) solution were prepared by initially dissolving $0.5 \mathrm{~g}$ of D-Phe or L-Phe, $50.43 \mathrm{~g}$ of DMSO and $2 \mathrm{ml}$ of TFA in $7.51 \mathrm{~g}$ of AA at $50^{\circ} \mathrm{C}$ for $2 \mathrm{~h}$ in the polymerization reactor. $37.72 \mathrm{ml}$ of AN was added to this solution and stirred for $5 \mathrm{~min}$. This was followed by the addition of $0.22 \mathrm{~g}$ of AIBN dissolved in $50 \mathrm{~g}$ of DMSO and purging with $\mathrm{N}_{2}$ for $5 \mathrm{~min}$. Then the copolymerization reaction was done for $6 \mathrm{~h}$ at 200 $\mathrm{rpm}$ and $60^{\circ} \mathrm{C}$ under a nitrogen atmosphere. After completion of the copolymerization reaction $100.86 \mathrm{~g}$ of DMSO were added into the polymer solution and stirred for about $20 \mathrm{~h}$ at $200 \mathrm{rpm}$ and $25^{\circ} \mathrm{C}$ in order to dilute the thick viscous solution. The prepared polymer solution was then kept in the vacuum oven for $24 \mathrm{~h}$ at $25^{\circ} \mathrm{C}$ temperature and $0.8 \mathrm{~atm}$ pressure to evaporate air bubbles and any moisture present.

Preparation of composite membrane comprised of DIBs and DIM (DCM) and composite membrane comprised of LIBs and LIM (LCM)

For this purpose $3 \%, \mathrm{w} / \mathrm{w}$, of already washed and freeze-dried DIBs or LIBs were dispersed into the prepared DIor LI-P (AN-co-AA) solutions respectively by vortexing with VORTEX-GENIES 2 (USA) for $10 \mathrm{~min}$ followed by 20 min of sonication with a NEXUL ultrasonicator. Entrapped air in the thick, viscous composite polymeric solution was removed by using a vacuum pump (Tokyo Rikakikai Co., Ltd. Japan) along with ultra sonication for 10-15 min. The composite solution was then cast onto the surface of glass plates with the help of an applicator (BYK Gardner 2021) and dipped into the distilled water for about $5 \mathrm{~min}$ at $25^{\circ} \mathrm{C}$ to obtain sheets of DCM and
LCM. The control membranes (DIMs and LIMs) were also prepared by the same method, but without the addition of DIBs and LIBs. The average thickness of the resultant composite membranes was $70 \sim 80 \mu \mathrm{m}$ while that of DIMs and LIMs was $40 \sim 50 \mu \mathrm{m}$.

Washing of the prepared composite membranes, control membranes and beads

The main extraction of the template molecules was done during coagulation step of the membrane formation. However, further washing of the prepared composite membranes and control membrane was carried out with a continuous exchange of 5\% acetic acid solution and distilled water for about $24 \mathrm{~h}$ on orbital shaker at 110 $\mathrm{rpm}$ and $25^{\circ} \mathrm{C}$ to remove the remaining template molecules. After completion of washing process both types of membranes were thoroughly washed with distilled water to remove the residual acetic acid. The prepared DIBs and LIBs were also washed excessively with 5\% acetic solution followed by distilled water till the removal of template molecules.

Physical characterization of the prepared composite and control membranes

Percent swelling ratio (SR\%) and Water holding capacity (WHC) of the prepared composite and control membranes were determined by the following equations.

$S R \%=\frac{\left(W_{w}-W_{e}\right)}{W_{e}} \times 100$

Where $W_{w}$ is the wet weight (g) of the membrane and $W_{e}$ is the dry weight (g) of the membrane at equilibrium. $W H C=W_{e}-W_{m}$

WHC $(\mathrm{g} / \mathrm{g})$ is measured as the amount $(\mathrm{g})$ of water adsorbed per unit mass $(\mathrm{g})$ of the membrane (composite or control), where $W_{m}$ is the freeze-dried weight ( $\mathrm{g}$ ) of the membrane.

\section{FE-SEM analysis}

A scanning electron microscopy (SEM) of the freeze-dried composite membranes was performed using a Hitachi S-4800 \& EDX-350 (Horiba) FE-SEM (Tokyo Japan). Samples were fixed in the brass holder and coated with $\mathrm{OsO}_{4}$ by VD HPC-ISW osmium coater (Tokyo, Japan) prior to FE-SEM observation.

\section{Ultrafiltration (UF)}

For conducting UF an assembly of 6 sheets of the prepared membranes was employed. The assembly was then fixed in a Millipore UF kit. The chiral separation ability of the DCM and LCM as well as DIMs and LIMs was investigated. An aqueous solution of $\mathrm{pH} 6$ containing $100 \mathrm{mg} \mathrm{L}^{-1}$ of $\mathrm{D}$, L-Phe (50 mg for each enantiomer) was passed through membranes in a UF kit driven by a pressure of $1 \mathrm{~atm} .20 \mathrm{ml}$ of the feed volume was used initially. Samples were collected from permeate and retentate after each $2 \mathrm{ml}$ of permeation. The permeation time for collection of each $2 \mathrm{ml}$ permeate sample was 2-3 min. For sampling, the filtration process was stopped each time. The time for taking each of the retentate samples was approximately $2 \mathrm{~min}$. 


\section{High performance liquid chromatography (HPLC) analysis}

The amounts of D-Phe and L-Phe were measured by analyzing the obtained permeate and retentate samples using high-performance liquid chromatograph equipped with an M930 solvent delivery pump and M720 UV absorbance detector (Young-Lin Instruments, Anyang, Korea). A TSK gel Enantio L2 column (Tosoh, Tokyo, Japan) with dimensions of $4.6 \mathrm{~mm} \times 250 \mathrm{~mm}$ was used.

From HPLC results various parameters of the prepared DCM, LCM and their control membranes were calculated by different equations as reported previously ${ }^{34}$ which are mentioned as following:

Amount of Phe adsorbed:

$Q=\frac{M_{o}-M_{p}-M_{r}}{W_{m}}$

Where $Q$ is the amount of D- or L-Phe adsorbed on the membrane $\left(\mathrm{mg} \mathrm{g}^{-1}\right)$, while $M_{o}, M_{p}$ and $M_{r}$ are the amounts of the D- or L-Phe (mg) in the feed solution, permeate and retentate, respectively.

Adsorption selectivity:

$\alpha_{a d s}=\frac{Q_{1}}{Q_{2}}$

Where $\alpha_{\text {ads }}$ is the adsorption selectivity, $Q_{1}$ and $Q_{2}$ are the amounts $\left(\mathrm{mg} \mathrm{g}^{-1}\right)$ of the template and the counter enantiomer adsorbed on the membrane, respectively.

Selective rejection of the solute:

$R=\left[\frac{\left(C_{r}-C_{o}\right) V_{r}}{\left(C_{o} \times V_{p}\right)}\right] \times 100$

Where $R$ is the rejection of $\mathrm{D}$ - or L-Phe; $V_{r}$ represents the volume $\left(\mathrm{m}^{-3}\right)$ of retentate; $V_{p}$ represents the volume $\left(\mathrm{m}^{-3}\right)$ of permeate and $C_{r}$ and $C_{o}$ are the concentrations $\left(\mathrm{mg} \mathrm{m}^{-3}\right)$ in the retentate and feed solution, respectively. The selective rejection $(R)$ is calculated in percentage hence the data obtained is represented as percent rejection and is mentioned as Rejection (\%).

Rejection selectivity of the membrane:

$\alpha_{r e j}=\frac{R_{1}}{R_{2}}$

Where $\alpha_{r e j}$ is the rejection selectivity, $R_{1}$ and $R_{2}$ are the rejection of the template and counter enantiomer by the membrane, respectively.

Permeability coefficient:

$P=\frac{f \partial}{C_{o}-C_{p}}$

Where $P$ is the permeability coefficient $\left(\mathrm{m}^{2} \cdot \mathrm{s}^{-1}\right), f$ is the flux $\left(\mathrm{mg} \cdot \mathrm{m}^{-2} \cdot \mathrm{s}^{-1}\right)$ calculated by dividing $M_{p}$ by unit area $\mathrm{A}\left(\mathrm{m}^{2}\right)$ of the membrane and time $\mathrm{T}(\mathrm{s}) ; \partial$ is the membrane thickness $(\mathrm{m})$; and $C_{p}$ is the concentration $\left(\mathrm{mg} \mathrm{m}^{-3}\right)$ of $\mathrm{D}$ - or L-Phe in permeate respectively.

Permselectivity:

$\alpha_{\text {perm }}=\frac{P_{1}}{P_{2}}$

Where $\alpha_{\text {perm }}$ is the permselectivity, $P_{1}$ and $P_{2}$ are the permeability coefficient $\left(\mathrm{m}^{2} \mathrm{~s}^{-1}\right)$ of the template and counter enantiomer.

\section{RESULTS AND DISCUSSION}

\section{Composite membrane morphology}

The structural and morphological study of the prepared DCM and LCM was done by SEM analyses. The SEM micrographs shown in Figure 1 (A-D) represent different aspects of the prepared composite membranes. Figure 1 (A) and Figure 1 (C) represent the nanoporous structure of DCM and LCM, respectively. The cross sectional morphology and a uniform dispersion of beads in the DCM and LCM matrix is shown in Figure 1 (B) and Figure 1 (D). The uniform dispersion of beads within the composite membrane matrix was achieved due to the suitable dispersion methods, appropriate sizes and spherical shapes of beads. Furthermore, the uniform dispersion of beads within the DCM and LCM matrix was made possible in a short time without using any surfactant or other chemical for dispersion purpose.

The packing of submicron/nanoscale beads inside the DCM and LCM matrix caused an increase in its thickness compared to non-composite membranes. Figure 1 (B) and (D) also clarify that the beads were uniformly dispersed within the composite membrane and had faced no damage during their dispersion and casting processes. The DCM and LCM maintained the basic three layered structure of the P (AA-co-AN) membranes prepared by phase inversion technique ${ }^{\mathbf{6}, \mathbf{2 5}, \mathbf{2 6}}$. Similarly, the surface topology and pore symmetry shown in Figure 1 (A) and (C) clarify that the DCM and LCM not only provided a large surface area, but it also maintained the nano-porous structure for active permeation of the enantiomers during UF.
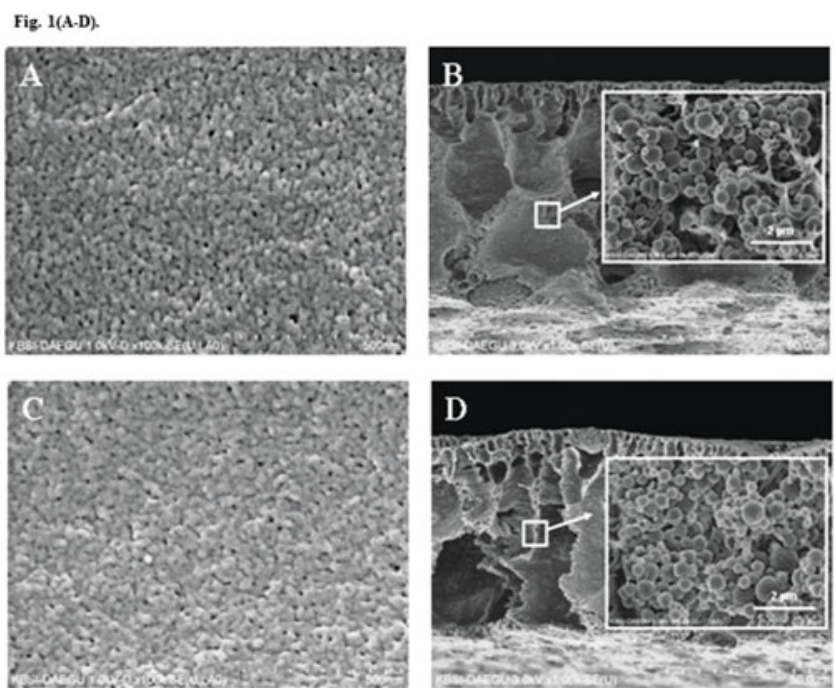

Figure 1. FE-SEM micrographs of the prepared DCM and LCM where (A) and (C) represent the nanoporous surface and (B) and (D) show the nanobeads distribution in the polymer matrix of DCM and LCM respectively

\section{Extraction of template}

The extraction of template molecules for getting specific binding sites in the polymer matrix is an important step in molecular imprinting ${ }^{35}$. In this study it was found that most of the template molecules (more than $73 \%$ ) were dissolved in water during the coagulation step of DCM and LCM preparation. The remaining template molecules 
were extracted with $5 \%$ acetic acid solution. Similarly, in case of DIMs and LIMs more than $69 \%$ of template molecules were removed during coagulation followed by washing with $5 \%$ acetic acid and distilled water for complete removal of template molecules. After complete washing a total of $88.68 \%$ and $89.25 \%$ of template molecules were extracted from DCM and LCM respectively, compared to $83.75 \%$ and $84.29 \%$ in the case of DIMs and LIMs respectively. The relatively higher extraction of template from the composite membrane matrix was due to the use of physico-mechanical processes during composite membrane preparations. These processes loosened the membrane structures and hence, the removal of the template was easier compared to non-composite membrane where no such process was applied.

\section{Physical characterization}

The WHC and SR\% of the prepared DCM and LCM were studied and compared with DIM and LIM respectively. DCM and LCM showed a high SR\% compared to their control membranes as clarified from Figure 2. The DCM and LCM had a greater WHC compared to their control. WHC of DCM and LCM were $0.161 \mathrm{~g} \mathrm{~g}^{-1}$ and $0.166 \mathrm{~g} \mathrm{~g}^{-1}$ respectively, compared to $0.146 \mathrm{~g} \mathrm{~g}^{-1}$ and $0.149 \mathrm{~g} \mathrm{~g}^{-1}$ for DIM and LIM respectively. The maximum SR\% values $387.55 \%$ and $395.53 \%$ for DCM and

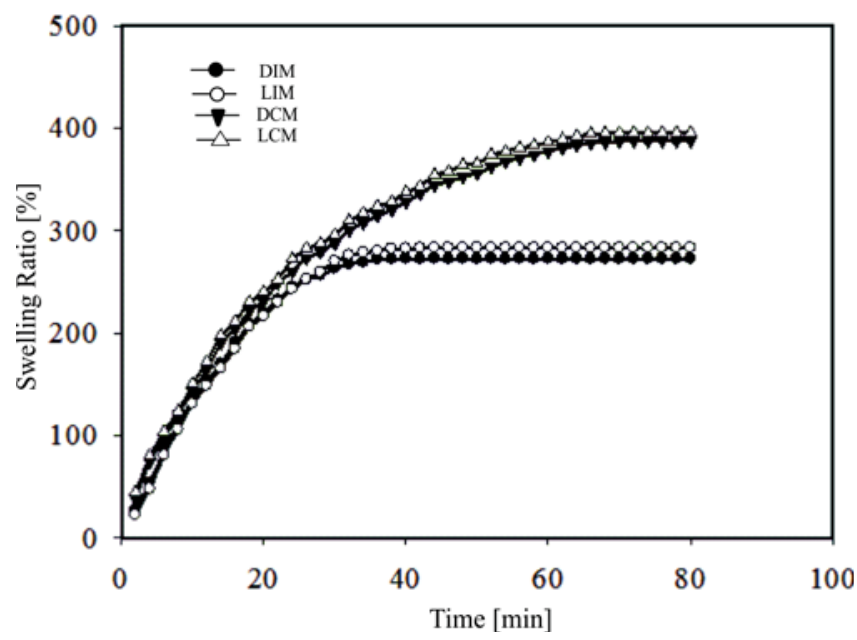

Figure 2. Percent swelling ratio with respect to time profile of DCM and LCM and DIM. For determining the swelling ratios, single sheets of DCM and LCM and DIMs were immersed in distilled water for $24 \mathrm{~h}$ at $25^{\circ} \mathrm{C}$ and its wet weight was determined followed by measuring the equilibrium dry weight at $25^{\circ} \mathrm{C}$. The difference in weights was used to calculate the percent swelling ratios
LCM respectively, were also much higher compared to $272.49 \%$ and $283.12 \%$ for DIM and LIM respectively.

All of these changes in the physical properties of DCM and LCM may be considered due to a large surface area, high thickness and an increase in the number of available binding sites (ionic groups) in the prepared DCM and LCM owing to the incorporation of imprinted submicron/nanoscale beads ${ }^{\mathbf{1 2}, \mathbf{3 6}, \mathbf{3 7}}$. The enhanced WHC, SR\% and binding capacity, support the fact that even after incorporation the imprinted submicron/nanoscale beads had performed their role actively inside the DCM and LCM matrix.

\section{Adsorption and adsorption selectivity}

The use of monodisperse MIP particles has great influence in enhancing the binding capacities of the MIMs ${ }^{\mathbf{1 2}}$. Several attempts for increasing the adsorption ability and selectivity of the MIP beads and MIP membranes were made as such or by incorporating the MIP particles into or on the non-MIP polymer membrane matrix ${ }^{\mathbf{1}}$ 19, 24-36. Similarly, in case of MIP composite membranes prepared by coating thin MIP film over the non-MIP support also showed good adsorptive behavior ${ }^{15}$. However, almost all of these composite systems used a non-MIP support and their operation time was much longer compared to the present composite system. Table 1 shows that after initial $2 \mathrm{ml}$ of permeation the adsorption capacity and selectivity of DCM and LCM were more than 37 to 60 times higher than that of control membranes (DIMs and LIMs respectively). Similarly, Table 1 also clarifies that their adsorption selectivity was also higher than the Phe imprinted beads. Figure 3 (A) shows that the maximum cumulative adsorption capacity of DCM for D-Phe and L-Phe were $1.970 \mathrm{mg} \mathrm{g}^{-1}$ and $1.781 \mathrm{mg} \mathrm{g}^{-1}$ (after $12 \mathrm{ml}$ of permeation). Similarly, Figure 4 (B) shows that the maximum adsorption capacity of LCM for D-Phe and L-Phe were $1.881 \mathrm{mg} \mathrm{g}^{-1}$ and $2.077 \mathrm{mg} \mathrm{g}^{-1}$ respectively. One evident cause for this much higher adsorption capacity was the applied pressure which made the access of template to the deeper sites of the incorporated beads within the composite membranes. UF was carried out at 1 atm gauge pressure and thus the adsorption capacity of incorporated beads in a composite membrane increased during UF as explained by different adsorption studies $^{\mathbf{3 6}, 37}$. However, there were also several other factors which made possible the high adsorption capacity of the composite membranes compared to the control membrane. The increase in the surface area ${ }^{27,37}$ and the availability of a large number of functional sites ${ }^{\mathbf{3 8}}$ due

Table 1. Comparative study of different parameters of DCM, LCM, DIMs and LIM after filtration of $12 \mathrm{ml}$ of a $100 \mathrm{mg} \mathrm{L^{-1 }}$ racemic mixture of phenylalanine through an assembly of 6 sheets of each at $1 \mathrm{~atm}$ applied pressure. The adsorption capacity and adsorption selectivity of DIBs and LIBs were calculated after shaking $0.1 \mathrm{~g}$ of DIBs and LIBs soaked in $2 \mathrm{ml}$ of $100 \mathrm{mg} \mathrm{ml}^{-1}$ of phenylalanine racemate solution

\begin{tabular}{|c|c|c|c|c|c|c|}
\hline \multirow[t]{2}{*}{ Type } & \multirow{2}{*}{$\begin{array}{l}\text { Adsorbed amount of } \\
\text { Phe }\left[\mathrm{mg} \cdot \mathrm{g}^{-1}\right]\end{array}$} & \multirow[t]{2}{*}{$\begin{array}{l}\text { Adsorption } \\
\text { selectivity }\end{array}$} & \multicolumn{2}{|c|}{$\begin{array}{c}\text { Permeability } \\
\text { Coefficient }\left[\mathrm{m}^{2} \cdot \mathrm{s}^{-1}\right]\end{array}$} & \multicolumn{2}{|c|}{$\begin{array}{c}\text { Rejection } \\
{[\%]}\end{array}$} \\
\hline & & & D-Phe & L-Phe & D-Phe & L-Phe \\
\hline DCM & 0.334 & 2.722 & $0.159 \times 10^{-5}$ & $0.211 \times 10^{-5}$ & 7.156 & 22.678 \\
\hline LCM & 0.365 & 2.983 & $0.179 \times 10^{-5}$ & $0.140 \times 10^{-5}$ & 26.282 & 6.223 \\
\hline DIM & 0.009 & 1.848 & $0.257 \times 10^{-5}$ & $0.151 \times 10^{-5}$ & 19.071 & 29.444 \\
\hline LIM & 0.006 & 1.977 & $0.149 \times 10^{-5}$ & $0.265 \times 10^{-5}$ & 29.914 & 18.963 \\
\hline${ }^{*}$ DIBs & 0.284 & 1.565 & $x^{2}$ & - & - & - \\
\hline *LIBs & 0.331 & 1.588 & - & - & - & - \\
\hline
\end{tabular}

* Batch adsorption was carried out at an atmospheric pressure (with no gauge pressure). 

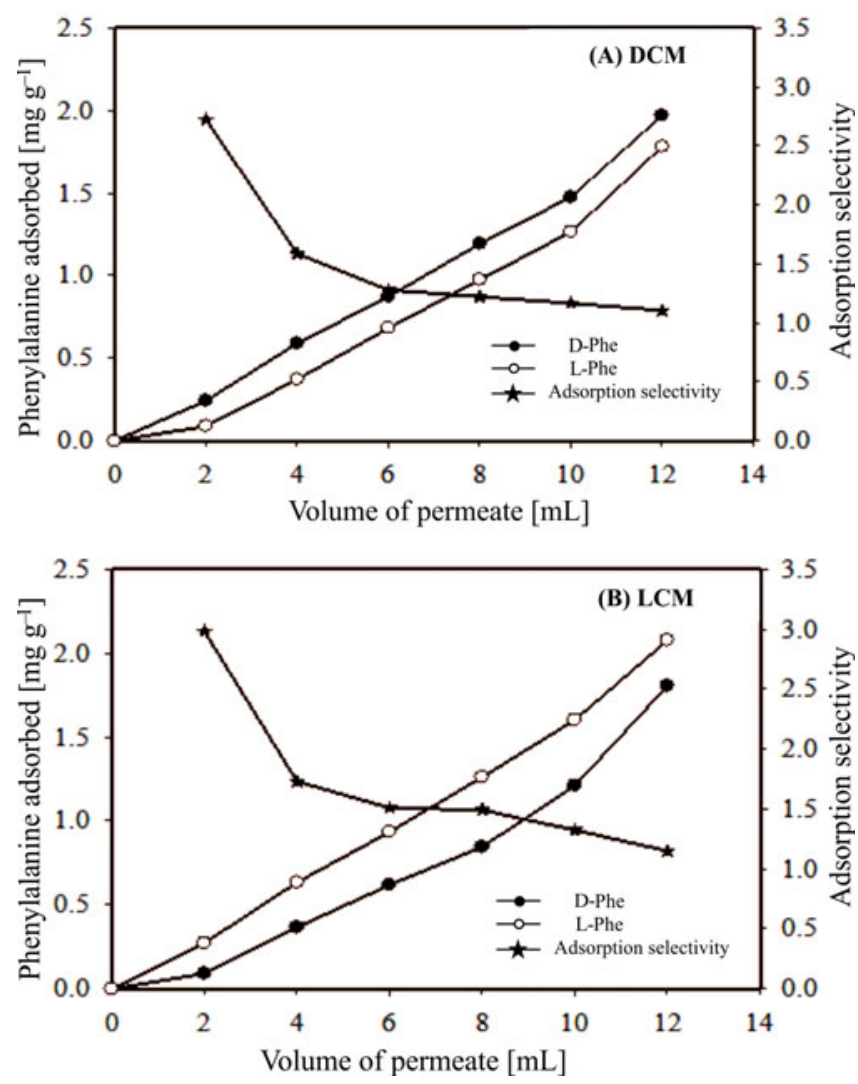

Figure 3. Specific adsorption and adsorption selectivity profiles of D- and L-Phe during filtration of $12 \mathrm{ml}$ of 100 mg L-1 racemic mixture of phenylalanine through an assembly of 6 sheets of (A) DCM and (B) LCM at $1 \mathrm{~atm}$ applied pressure

to incorporated imprinted beads and the formation of suitable environment within the composite membranes matrix were the various causes for achieving the higher adsorption capacity ${ }^{\mathbf{1}, \mathbf{2 6}, \mathbf{2 6}}$.

The adsorption selectivity of DCM and LCM has also been much improved compared to control membranes and beads. Table 1 shows that the maximum adsorption selectivity achieved after initial $2 \mathrm{ml}$ of permeation were 2.722 and 2.983 for DCM and LCM respectively, compared to 1.848 and 1.977 for DIM and LIM respectively. Figure 3 (A) and (B) show that initially the adsorption selectivity was higher, but then decreased gradually with an increase in permeation volume. This may be due to the fact that with an increase in permeation volume most of the selective binding sites were occupied ${ }^{39}$ owing to which the specific adsorption of template molecules ceased. Besides this, the increase in concentration of the retentate solution due to the rejection phenomenon may also be considered as a major factor responsible for a decrease in adsorption selectivity ${ }^{34}$ with an increase in permeation volume.

This study shows that LCM has the highest value of adsorption capacity and adsorption selectivity which was due to the high adsorption capacity and selectivity of LIM and the incorporated LIBs. Hence, it is evident from this study that the combining MIP formats have great influence on the selective adsorption performance of the resultant composite system.

\section{Rejection and rejection selectivity}

The membranes performance cannot be analyzed perfectly by studying only its separation ability, but their re-
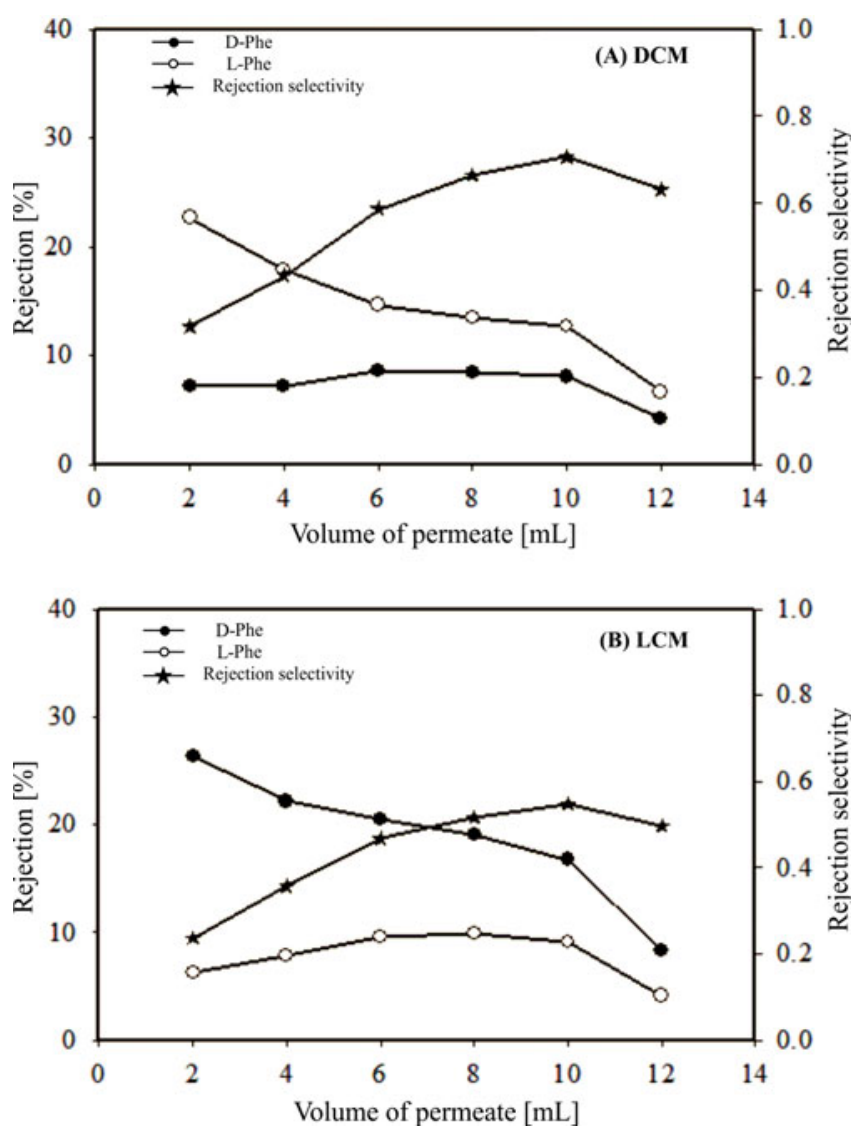

Figure 4. Rejection and rejection selectivity profiles of D- and L-Phe during filtration of $12 \mathrm{ml}$ of $100 \mathrm{mg} \mathrm{L}-1$ racemic mixture of phenylalanine through an assembly of 6 sheets of (A) DCM and (B) LCM at $1 \mathrm{~atm}$ applied pressure

jection and flux properties should also be considered ${ }^{\mathbf{4 0 , 4 1}}$. Table 1 shows the rejection study of the DCM and LCM assembly which indicates that the percent rejection for both of the enantiomers is lower compared to DIMs and LIMs. This decrease in percent rejection for both of the enantiomers may be considered due to high adsorption on the surface as well as inside the composite membranes system as the imprinted membranes have binding sites on the surface as well as inside the matrix ${ }^{15,16}$. The comparatively less rejection may be considered suitable for enhanced adsorption and extraction purposes because this enhances the chances of binding sites accessibility and have a positive effect on permeation. Figure 4 (A) and (B) show that the percent rejection was decreased with increase in permeation volume. The cause for this decrease in rejection is the membranes stretch and pore deformation phenomena under the applied pressure ${ }^{\mathbf{3 4 , 4 2}}$ with an increase in permeation volume.

Rejection selectivity value is much lower than 1 which indicates the greater rejection of counter enantiomer compared to template molecules. The imprinted composite membrane matrix having the specific gates for template molecules hindered the penetration of counter enantiomer, which resulted in their higher rejection compared to the template molecules ${ }^{34}$.

\section{Permeability coefficient and permselectivity}

The permeability coefficient and permselectivity values of a membrane system may give an idea about its separation ability ${ }^{\mathbf{1 2}, 38}$. From Figure 5 (A) and (B) it is clear that the value of permselectivity is less than 1 . This is because 

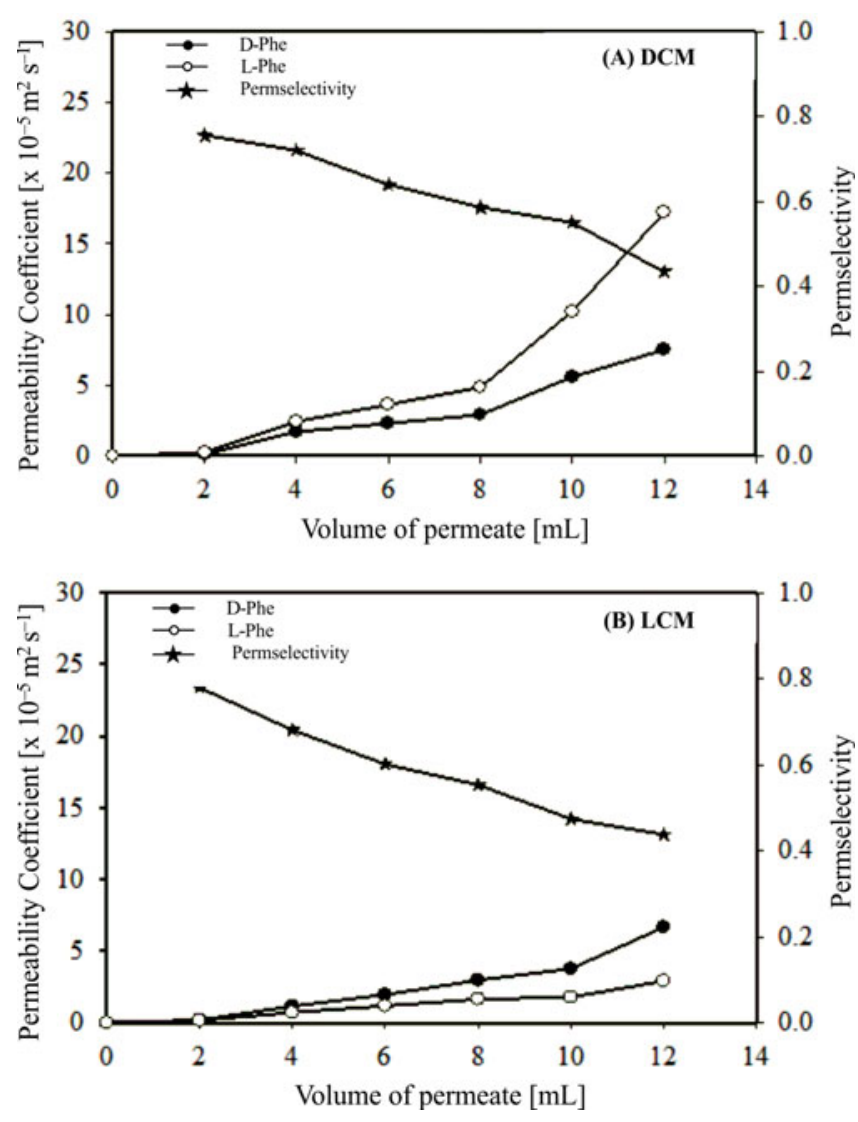

Figure 5. Permeability coefficient and permselectivity profiles of D- and L-Phe during filtration of $12 \mathrm{ml}$ of 100 mg L-1 racemic mixture of phenylalanine through an assembly of 6 sheets of (A) DCM and (B) LCM at $1 \mathrm{~atm}$ applied pressure

the DCM and LCM selectivity adsorbed higher amount of template compared to counter enantiomer owing to incorporated DIBs and LIBs respectively. This permselectivity study clarifies that the greater selective adsorption of template molecules caused by the incorporated DIBs and LIBs as well as the membrane matrix of DCM and LCM respectively. Figure 5 (A) and (B) clarify that the permeability coefficient values for both the enantiomers increased with an increase in permeation volume, which may be due to membrane structural distortion and pore enlargement ${ }^{34}$ with an increase in permeation volume. Ulbrich $t^{12}$ reported that the increase in permeability is due to membrane swelling as a consequence of template binding to imprinted sites which is also a considerable reason for this increase.

The permselectivity value of DCM and LCM was lower than 1 which indicates the greater adsorption of template molecules compared to counter enantiomer ${ }^{30}$. The greater adsorption of template molecules by composite membranes resulted in a higher permeation of counter enantiomers. It clarified that the phenomenon of retarded transport caused by incorporated DIBs and LIBs in the DCM and LCM respectively was greater compared to the facilitated transport maintained by the composite membranes matrix. Hence, it is concluded that the higher selective adsorption and lower rejection had imposed a great influence on selective permeation properties of the composite membranes.

\section{Conclusions}

Composite MIP membranes (DCM and LCM) were prepared by the phase inversion method after dispersing DIBs and LIBs into the DI-P (AN-co-AA) and LI-P (AN-co-AA) solutions respectively by a simple physico-mechanical process. FE-SEM analysis showed the nanoporous surface morphology and uniform distribution of Phe imprinted beads into the Phe imprinted composite membrane. The DCM and LCM showed a greater adsorption capacity and adsorption selectivity compared to control membranes and beads. Similarly, the as-prepared imprinted composite membranes showed less percent rejection and inverse permselectivity compared to their respective control membrane. The adsorption capacity and adsorption selectivity of LCM were higher than that of DCM. In short, the composite membranes comprised of imprinted beads and membranes selective for same template molecule followed the selective adsorption which resulted in the retarded transport of template molecules. Due to having high adsorption capacity and selectivity, these composite systems will be useful in future for separation, purification, drug delivery systems and in various biomedical applications.

\section{Nomenclature}

D-Phe D-Phenylalanine

L-Phe L-Phenylalanine

DCM Composite membrane comprised of DIBs and DIM

LCM Composite membrane comprised of LIBs and LIM

DIBs D-Phe imprinted P (MMA-co-EGDMA) submicron/nanoscale beads

LIBs L-Phe imprinted P (MMA-co-EGDMA) submicron/nanoscale beads

DI-P (AN-co-AA) D-Phe incorporated poly (acrylonitrile-co-acrylic acid)

LI-P (AN-co-AA) L-Phe incorporated poly (acrylonitrile-co-acrylic acid)

DIM D-Phe imprinted P (AN-co-AA) membrane

LIM L-Phe imprinted P (AN-co-AA) membrane

UF ultrafiltration

$A \quad$ effective area of the membrane $\left[\mathrm{m}^{2}\right]$

$C_{o} \quad$ concentration of D- or L-Phe in the feed solution $\left[\mathrm{mg} \mathrm{m}^{-3}\right]$

$C_{p} \quad$ concentration of D- or L-Phe in permeate [mg $\mathrm{m}^{-3}$ ]

$C_{r} \quad$ concentrations of D- or L-Phe in the retentate [mg m${ }^{-3}$ ]

$f \quad$ flux of D- or L-Phe $\left[\mathrm{mg} \mathrm{m}^{-2} \mathrm{~s}^{-1}\right]$

$M_{o} \quad$ amount of D- or L-Phe in the feed solution [mg]

$M_{p} \quad$ amount of D- or L-Phe in the permeate [mg]

$M_{r} \quad$ amount of D- or L-Phe in the retentate [mg]

$P \quad$ permeability coefficient of the D- or L-Phe $\left[\mathrm{m}^{2} \mathrm{~s}^{-1}\right]$

$P_{1} \quad$ permeability coefficient of the template $\left[\mathrm{m}^{2} \mathrm{~s}^{-1}\right]$

$P_{2} \quad$ permeability coefficient of the counter enantiomer $\left[\mathrm{m}^{2} \mathrm{~s}^{-1}\right]$

$Q \quad$ amount of the D- or L-Phe adsorbed on the membrane $\left[\mathrm{mg} \mathrm{g}^{-1}\right]$ 
$Q_{1} \quad$ amount of the template adsorbed on the membrane $\left[\mathrm{mg} \mathrm{g}^{-1}\right.$ ]

$Q_{2} \quad$ amount of the counter enantiomer adsorbed on the membrane $\left[\mathrm{mg} \mathrm{g}^{-1}\right]$

$R \quad$ the percent rejection of the D- or L-Phe

$R_{1} \quad$ rejection of the template by the membrane

$R_{2} \quad$ rejection of the counter enantiomer by the membrane

SR\% percent swelling ratio

$t$ time required for a solution to pass through the membrane [s]

$V_{r} \quad$ volume of the retentate $\left[\mathrm{m}^{-3}\right]$

WHC water holding capacity

$W_{e} \quad$ dry weight of the membrane at equilibrium [g]

$W_{m} \quad$ freeze dried weight of the membrane $[\mathrm{g}]$

$W_{w} \quad$ wet weight of the membrane [g]

$\partial$ the membrane thickness [m]

$\alpha_{\text {ads }}$ adsorption selectivity

$\alpha_{\text {perm }}$ permselectivity

$\alpha_{\text {rej }}$ rejection selectivity

\section{LITERATURE CITED}

1. Takeda, K. \& Kobayashi, T. (2006). Hybrid molecularly imprinted membranes for targeted bisphenol derivatives. J. Membr. Sci. 275(1), 61-9. DOI: 10.1016/j.memsci.2005.09.004. 2. Sharma, P.S., Iskierko, Z., Pietrzyk-Le, A., D’Souza, F. \& Kutner, W. (2015). Bioinspired intelligent molecularly imprinted polymers for chemosensing: A mini review. Electrochem. Commun. 50, 81-87. DOI:10.1016/j.elecom.2014.11.019.

3. Lv, Y.K., Zhang, J.Q., He, Y.D., Zhang, J. \& Sun, H.W. (2014). Adsorption-controlled preparation of molecularly imprinted hybrid composites for selective, extraction of tetracycline residues from honey and milk. New. J. Chem. 38, 802-808. DOI: $10.1039 / \mathrm{C} 3 \mathrm{NJ} 00962 \mathrm{~A}$.

4. Park, J.K. \& Seo, J.I. (2002). Characteristics of phenylalanine imprinted membrane prepared by the wet phase inversion method. Korean J. Chem. Eng. 19(6), 940-8. DOI: 10.1007/ BF02707215.

5. Park, J.K. \& Kim, S.J. (2004). Separation of phenylalanine by ultrafiltration using D-Phe imprinted polyacrylonitrile-poly (acrylic acid)-poly (acryl amide) terpolymer membrane. Korean j. Chem. Eng. 21(5), 994-8. DOI: 10.1007/BF02705583.

6. Scorrano, S., Mergola, L., Bello, M.P.D., Lazzoi, M.R., Vasapollo, G. \& Sole, R.D. (2015). Molecularly imprinted composite membranes for selective detection of 2-deoxyadenosine in urine samples. Int. J. Mol. Sci. 16, 13746-13759. DOI: $10.3390 / \mathrm{ijms} 160613746$.

7. Park, J.K., Kim, S.J. \& Lee, J.W. (2003). Adsorption selectivity of phenylalanine imprinted polymer prepared by the wet phase inversion method. Korean J. Chem. Eng. 20(6), 1066-1072. DOI: 10.1007/BF02706937.

8. Tasselli, F., Donato, L. \& Drioli, E. (2008). Evaluation of molecularly imprinted membranes based on different acrylic copolymers. J. Membr. Sci. 320(1), 167-72. DOI: 10.1016/j. memsci.2008.03.071.

9. Wu, Y., Liu, X., Meng, M., Li, P., Yan, M., Wei, X., Li, H., Yan, Y. \& Li, C. (2015). Bio-inspired adhesion: Fabrication of molecularly imprinted nanocomposite membranes by developing a hybrid organic-inorganic nanoparticles composite structure. J. Membr. Sci. 490, 169-178. DOI: 10.1016/j.memsci.2015.04.023. 10. Zhou, Y., Zhou, T., Jin, H., Jing, T., Song, B., Zhou, Y., Mei, S. \& Lee, Y.I. (2015). Rapid and selective extraction of multiple macrolide antibiotics in foodstuff samples based on magnetic molecularly imprinted polymers. Talanta 137, 1-10. DOI: 10.1016/j.talanta.2015.01.008.
11. Algieri, C., Drioli, E., Guzzo, L. \& Donato, L. (2014). Biomimetic sensors based on molecularly imprinted membranes. Sensors 14, 13863-13912. DOI: 10.3390/s140813863.

12. Ulbricht, M. (2004). Membrane separations using molecularly imprinted polymers. J. Chromatogr. B. 804(1), 113-25. DOI: 10.1016/j.jchromb.2004.02.007.

13. Jantarat, C., Tangthong, N., Songkro, S., Martin, G.P. \& Suedee, R. (2008). S-Propranolol imprinted polymer nanoparticle-on-microsphere composite porous cellulose membrane for the enantioselectively controlled delivery of racemic propranolol. Int. J. Pharm. 349(1), 212-25. DOI: 10.1016/j. ijpharm.2007.07.030.

14. Hilal, N., Kochkodan, V., Al-Khatib, L. \& Busca, G. (2002). Characterization of molecularly imprinted composite membranes using an atomic force microscope. Surf. Inter. Anal. 33(8), 672-5. DOI: 10.1002/sia.1434.

15. Wang, P., Hu, W. \& Su, W. (2008). Molecularly imprinted poly (methacrylamide-co-methacrylic acid) composite membranes for recognition of curcumin. Anal. Chim. Acta 615(1), 54-62. DOI: 10.1016/j.aca.2008.03.040.

16. Laroche, M., Pukall, R. \& Ulber, R. (2003). Gewinnung und Charakterisierung einer L-Serindehydratase aus dem marinen Bakterium Paracoccus seriniphilus zum Aufbau bioanalytischer Systeme. Chemie Ingenieur Technik 75(1-2), 146-9. DOI: 10.1002/cite.200390012.

17. Son, S.H. \& Jegal, J. (2007). Chiral separation of D, Lserine racemate using a molecularly imprinted polymer composite membrane. J. Appl. Poly. Sci. 104(3), 1866-72. DOI: 10.1002/app. 25845.

18. Yoshimatsu, K., Ye, L., Lindberg, J. \& Chronakis, I.S. (2008). Selective molecular adsorption using electrospun nanofiber affinity membranes. Biosens. Bioelectron. 23(7), 1208-15. DOI: 10.1016/j.bios.2007.12.002.

19. Fakirov, S., Bhattacharyya, D. \& Shields, R. (2008). Nanofibril reinforced composites from polymer blends. Colloids Surf. A Physicochem. Eng. Asp. 313, 2-8. DOI: 10.1016/j. colsurfa.2007.05.038.

20. Shah, N., Ha, J.H., Ul-Islam, M. \& Park, J.K. (2011). Highly improved adsorption selectivity of L-phenylalanine imprinted polymeric submicron/nanoscale beads prepared by modified suspension polymerization. Korean J. Chem. Eng. 28(9), 1936-44. DOI: 10.1007/s11814-011-0043-3.

21. Huangfu, F., Wang, B., Shan, J. \& Zhang, Z. ( 2013). Enantioselective analysis of naproxen using chiral molecular imprinting polymers based thin-layer chromatography. e-Polymers. 13(1), 180-188. DOI: 10.1515/epoly-2013-0117.

22. Tiwari, M.P. \& Prasad, A. (2015). Molecularly imprinted polymer based enantioselective sensing devices: A review. Anal. Chim. Acta 853, 1-18. DOI: 10.1016/j.aca.2014.06.011.

23. Yoshimi, Y. \& Ishii, N. (2015). Improved gate effect enantioselectivity of phenylalanine-imprinted polymers in water by blending crosslinkers. Anal. Chim. Acta 862, 77-85. DOI: 10.1016/j.aca.2015.01.001.

24. Lehmann,, M. Brunner, H. \& Tovar, G. (2002). Selective separations and hydrodynamic studies: a new approach using molecularly imprinted nanosphere composite membranes. Desalination 149(1), 315-21. DOI: 10.1016/S0011-9164(02)00754-3. 25. Borrelli, C. Barsanti, S. Silvestri, D. Manesiotis, P. Ciardelli, G. \& Sellergren, B. (2011). Selective depletion of riboflavine from beer using membranes incorporating imprinted polymer particles. J. Food Proc. Pres. 35(1), 112-128. DOI: 10.1111/j.1745-4549.2009.00464.x.

26. Silvestri, D., Barbani, N., Cristallini, C., Giusti, P. \& Ciardelli, G. (2006). Molecularly imprinted membranes for an improved recognition of biomolecules in aqueous medium. J. Membr. Sci. 282(1), 284-295. DOI: 10.1016/j.memsci.2006.05.031.

27. Faizal, C.K.M. \& Kobayashi, T. (2008). Tocopherol-targeted membrane adsorbents prepared by hybrid molecular imprinting. Polym. Eng. Sci. 48(6), 1085-1093. DOI: 10.1002/pen.21053. 
28. Roper, D.K. \& Lightfoot, E.N. (1995). Separation of biomolecules using adsorptive membranes. J. Chromatogr. A. 702(1), 3-26. DOI: 10.1016/0021-9673(95)00010-K.

29. Piletsky, S., Panasyuk, T., Piletskaya, E., Nicholls, I.A. \& Ulbricht, M. (1999). Receptor and transport properties of imprinted polymer membranes-a review. J. Membr. Sci. 157(2), 263-278. DOI: 10.1016/S0376-7388(99)00007-1.

30. Suedee, R., Bodhibukkana, C., Tangthong, N., Amnuaikit, C., Kaewnopparat, S. \& Srichana, T. (2008). Development of a reservoir-type transdermal enantioselective-controlled delivery system for racemic propranolol using a molecularly imprinted polymer composite membrane. J. Cont. Rel. 129(3), 170-8. DOI: 10.1016/j.jconrel.2008.05.001.

31. Kubo, T., Arimura, S., Tominaga, Y., Naito, T. Hosoya, K. \& Otsuka, K. (2015). Molecularly imprinted polymers for selective adsorption of lysozyme and cytochrome c using a PEG-based hydrogel: selective recognition for different conformations due to $\mathrm{pH}$ conditions. Macromolecules 48, 4081-4087. DOI: 10.1021/acs.macromol.5b00834.

32. Khan, H., Khan, T. \& Park, J.K. (2008). Separation of phenylalanine racemates using d-phenylalanine imprinted microbeads as HPLC stationary phase. Sep. Purif. Technol. 62(2), 363-369. DOI: 10.1016/j.seppur.2008.02.011.

33. Khan, H. \& Park, J.K. (2006). The preparation of D-phenylalanine imprinted microbeads by a novel method of modified suspension polymerization. Biotechnol. Bioprocess Eng. 11(6), 503-509. DOI: 10.1007/BF02932074.

34. Ul-Haq, N. Khan, T. \& Park, J.K. (2008). Enantioseparation with D-Phe- and L-Phe-imprinted PAN-based membranes by ultrafiltration. J. Chem. Tech. Biot. 83(4), 524-533. DOI: $10.1002 /$ jctb.1827.

35. Ellwanger, A., Berggren, C., Bayoudh, S., Crecenzi, C., Karlsson, L. \& Owens, P.K. (2001). Evaluation of methods aimed at complete removal of template from molecularly imprinted polymers. Analyst 126(6), 784-92. DOI: 10.1039/B009693H.

36. Bhatia, S., Tran, K., Nguyen, T. \& Nicholson, D. (2004). High-pressure adsorption capacity and structure of $\mathrm{CO} 2$ in carbon slit pores: theory and simulation. Langmuir 20(22), 9612-9620. DOI: 10.1021/la048571i.

37. Li, K., Olson, D.H., Lee, J.Y., Bi, W., Wu, K. \& Yuen, T. (2008). Multifunctional microporous MOFs exhibiting gas/ hydrocarbon adsorption selectivity, separation capability and three-dimensional magnetic ordering. Adv. Funct. Mater. 18(15), 2205-2214. DOI: 10.1002/adfm.200800058.

38. Hilal, N., Kochkodan, V., Busca, G., Kochkodan, O. \& Atkin, B. (2003). Thin layer composite molecularly imprinted membranes for selective separation of cAMP. Sep. Purif. Technol. 31(3), 281-289. DOI: 10.1016/S1383-5866(02)00205-8. 39. Yoshikawa, M., Ooi, T. \& Izumi, J.I. (2001). Novel membrane materials having EEE derivatives as a chiral recognition site. Eu. Polym. J. 37(2), 335-342. DOI: 10.1016/S0014-3057(00)00121-X.

40. Geens, J., Hillen, A., Bettens, B., Van der Bruggen, B. \& Vandecasteele, C. (2005). Solute transport in non-aqueous nanofiltration: effect of membrane material. J. Chem. Tech. Biot. 80(12), 1371-1377. DOI: 10.1002/jctb.1337.

41. Székely, G., Valtcheva, I.B., Kim, J.F. \& Livingston, A.G. (2015). Molecularly imprinted organic solvent nanofiltration membranes-Revealing molecular recognition and solute rejection behavior. React. Funct. Polym. 86, 215-224. DOI: 10.1016/j.reactfunctpolym.2014.03.008.

42. Park, J.K., Chang, H.N., Park, J.H. \& Earmme Y.Y. (1986). Direction-dependent flux anomalies in asymmetric reverseosmosis membranes. A theoretical analysis. Ind. Eng. Chem. 25(2), 189-195. DOI: 10.1021/i100022a003. 